\section{KDOQI update of hemoglobin target}

National Kidney Foundation Kidney Disease Outcomes Quality Initiative (KDOQI) clinical practice guidelines for anemia in chronic kidney disease (CKD) published in May 2006 included recommendations for hemoglobin $(\mathrm{Hb})$ targets in patients receiving erythropoiesis-stimulating agents (ESAs). These recommendations were based on analysis of a systematic review and randomized controlled trials. As one trial published in 2005 was not included in the analysis, however, and because five more randomized controlled trials have now been published, the guidelines have been updated.

The KDOQI Anemia Work Group re-examined all the available evidence on $\mathrm{Hb}$ targets, and updated two clinical practice recommendations and one clinical practice guideline on $\mathrm{Hb}$ targets. These updates apply to both patients who are and those who are not receiving concomitant iron therapy. One updated recommendation (2.1.1) states that potential benefits (e.g. improvements in quality of life and avoidance of transfusion) and risks (e.g. life-threatening adverse events) should be considered when selecting the $\mathrm{Hb}$ target and the $\mathrm{Hb}$ level at which ESA therapy should be initiated in individual patients. The other updated recommendation (2.1.2) was that the $\mathrm{Hb}$ target should usually be within the range $11.0-12.0 \mathrm{~g} / \mathrm{dl}(110-120 \mathrm{~g} / \mathrm{l})$ in dialysis patients and nondialysis patients with CKD who are receiving ESAs. Both updated recommendations were considered fully applicable to children. The updated guideline (2.1.3) states that the $\mathrm{Hb}$ target should not be more than $13.0 \mathrm{~g} / \mathrm{dl}(130 \mathrm{~g} / \mathrm{l})$ in dialysis patients and nondialysis patients with CKD receiving ESAs; this guideline is also applicable to children, but might need modification when more data become available.

Original article KDOQI (2007) KDOQI Clinical Practice

Guideline and Clinical Practice Recommendations for anemia in chronic kidney disease: 2007 update of hemoglobin target. Am J Kidney Dis 50: 471-530

\section{A diagnostic and treatment algorithm for dense deposit disease}

Dense deposit disease (DDD; also known as membranoproliferative glomerulonephritis type II) has a prevalence of just 2-3 cases per million individuals, and as a result it has been difficult to evaluate potential therapies in randomized controlled trials. Recently, Smith and colleagues have used available animal, genetic and molecular data to develop a diagnostic and treatment algorithm for the disease.

The authors recommend that all patients with biopsy-proven DDD should be assayed for C3 nephritic factor (C3NeF; an autoantibody against C3 convertase), tested for serum markers of complement activity, and screened for mutation of the factor $\mathrm{H}$ gene, $\mathrm{CFH}$. These tests are intended to evaluate abnormalities in the alternative complement pathway. If the C3NeF assay is positive, plasma exchange or infusion should be considered, as should administration of anti$B$-cell agents such as rituximab. If a pathologic $\mathrm{CFH}$ mutation is found, plasma infusion should be performed to provide functional factor $\mathrm{H}$. Depending on pathophysiology, administration of eculizumab (an anti-C5 antibody) or sulodexide (a heparanase inhibitor) can be considered. More generally, nonspecific treatments, including aggressive blood pressure control and reduction of proteinuria with angiotensin-converting-enzyme inhibitors and angiotensin-receptor blockers, might slow progression of renal damage. Treatment success should be defined as retardation of progressive kidney dysfunction, and secondarily as normalization of alternative complement pathway activity.

Reporting of DDD treatments and outcomes to the DDD Outcomes Database, a resource available to all health-care personnel at http://genome.uiowa.edu/ddd, will facilitate evidence-based management of this rare disorder.

Original article Smith RJH et al. (2007) New approaches to the treatment of dense deposit disease. J Am Soc Nephrol 18: $2447-2456$

\section{Frequent nocturnal hemodialysis improves outcomes compared with conventional hemodialysis}

Regression of left ventricular (LV) hypertrophy is associated with a decreased incidence of cardiovascular events, and could be targeted as a means of alleviating the high burden of cardiovascular-related deaths in the hemodialysis population. Results of a randomized controlled 